\title{
Strategic plan for the management of COVID-19 in an obstetrics department
}

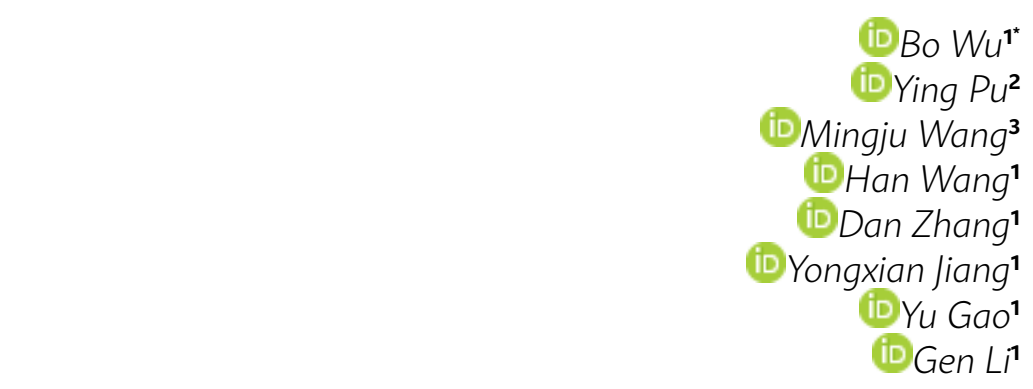

1. Department of Pharmacy, Chengdu Women's and Children's Central Hospital, School of Medicine, University of Electronic Science and Technology of China, Chengdu 611731, China. 2. Obstetrics Department, Chengdu Women's and Children's Central Hospital, School of Medicine, University of Electronic Science and Technology of China, Chengdu 611731, China. 3. Department of Neurology, Chengdu Fifth People's Hospital, Chengdu 611130, China.

KEYWORDS: Pregnant women. Coronavirus Infections. Obstetrics and Gynecology Department, Hospital.

The Coronavirus Disease 2019 (COVID-19) pandemic, caused by the Severe Acute Respiratory Syndrome Coronavirus 2 (SARS-CoV-2), has developed into a worldwide crisis for this generation'. In March 2020, the World Health Organization (WHO) declared the COVID-19 to be a world pandemic. Up to May 20, a total of 4,904,805 people had been confirmed to be affected by COVID-19, and 320,474 people have died from this crisis ${ }^{2}$.

Pregnant women are vulnerable during infectious disease outbreaks due to their special physiological state ${ }^{3}$. Because of physiological changes in the immune and cardiopulmonary systems, pregnant women are more likely to develop severe illness after respiratory infection ${ }^{4}$. According to previous research, SARS-CoV and MERS-CoV (highly homologous to SARS-CoV-2) are both known to be responsible for severe complications during pregnancy, including the need for endotracheal intubation, admission to an intensive care unit (ICU), renal failure, and death ${ }^{5}$. Up to now, no study showed that pregnant women are more likely to be infected with COVID-19, and pregnancy outcomes are worse in diagnosed patients, and interrelated research is very inadequate $e^{6}$. Other than the maternal adverse effects of COVID-19, its potential impact on fetal and neonatal outcomes has not been ruled out either. Therefore, standard procedures are urgently needed to avoid hospital-related infection and guide the management of pregnant patients. Here, we proposed a strategic plan for the management of COVID-19 outbreaks in Obstetrics Departments, primarily focusing on the prevention and control strategies of viral infection. 


\section{EDUCATION}

Medical staff should be kept up to date with the latest information about COVID-19 on pregnant women (clinical features, complications, diagnosis, therapies, maternal and fetal outcomes, etc.). Education about COVID-19 is needed for pregnant patients and their caregivers. Patient education should include methods and timing of hand hygiene, respiratory hygiene, coughing etiquette, selection and use of mask, the transmission routes and clinical manifestations of COVID-19, and patients should be advised to stay at home and avoid densely populated areas. Besides, pregnant women should be informed to seek medical advice in case of fever, cough, weak, dyspnea. Mothers and carers should be educated in the selection and use of personal protective equipment (PPE), and how to put it on and take it off correctly.

\section{Establishing a COVID-19 expert committee}

A COVID-19 expert committee should be established in medical institutions for decision-making in multidisciplinary consultation meetings. Committee members should include: an obstetrician, respiratory physicians, infectious disease physicians, physicians from ICU, radiologists, clinical pharmacists, and medical staff from hospital infection-control departments.

\section{Regional management strategy}

To prevent cross-infection in the hospital, a regional management strategy should be used. Both employee and patient channels need to be built. Meanwhile, medical staff should wear gowns, masks, hats, and medical latex gloves when in contact with patients in the outpatient department or wards. Pre-examination and triage should be done in the entrance of the patient channel, and a special channel needs to be built for the transportation of patients with a suspicious epidemiological history or related symptoms.

Four zones should be created in the hospital to prevent cross-infection and to screen potentially infected patients. Medical staff and patients are not allowed to enter other zones without permission. Zone 1 (surveillance and screening) is for surveilling and screening subjects who are viewed by a expert as a patient potentially infected with SARS-CoV-2; each patient is isolated in a single room. Zone 2 (suspected quarantine) is for the suspected cases of COVID-19; each patient is isolated in a single room. Zone 3 (COVID-19 confirmed quarantine) is for confirmed COVID-19 cases. If the hospital does not have the necessary conditions to receive confirmed patients, then patients should be transferred immediately to specialized hospitals with the capacity for treatment. Zone 4 (obstetric ward) is for pregnant patients who do not have COVID-19. Personal protective equipment (PPE) should be provided in all zones, such as gowns, masks, eye shields, gloves, shoe covers, and hats. The printed procedures should be posted up in the workplace to remind the medical staff of when and how to use PPE.

\section{Antenatal care management}

Intrauterine pregnancy confirmation and antenatal care are essential for pregnant women. But we should reduce hospital visits and cancel part of the check-ups to avoid further spread of the epidemic. Patients need to connect with an obstetrician online first, and then determine whether it is necessary to cancel the hospital visit and routine check-up during pregnancy. Based on complications, clinical manifestation, adverse pregnancy history, and other risk factors, the obstetrician should give their expert advice on a hospital visit and routine check-up. The following check-ups are recommended: 1) in the first trimester, ultrasonographic examination to confirm pregnancy after 6 weeks of amenorrhea is advised. Ultrasonographic examination of the nuchal translucency (NT) along with blood pressure, fasting plasma glucose, ECG, ABO, and Rh blood group test, blood routine examination, routine urine test, and fetal heart rate monitoring are advised to be done in one visit between 11 to 13 weeks and 6 days of gestation; 2) in the second trimester, down's syndrome prenatal screening or non-invasive prenatal testing (NIPT) from cell-free fetal DNA are recommended to be done between 16 and $16+6$ gestational weeks. Oral glucose tolerance testing (OGTT) along with fetal congenital malformations screening (by four-dimensional color Doppler ultrasound combined with system-B ultrasound) should be done in one visit between 24 to $24+6$ weeks; 3 ) in the third trimester, a routine visit every 2 weeks should be changed to 3 weeks. Ultrasonographic examination and Group B streptococci (GBS) test are recommended to be done in one visit between 30 to 32 weeks and another visit between 35 to 37 weeks; 4) Fetal heart rate monitoring is advised, which can be done at home if rental remote monitoring device is available; 5) Certain surveillances such as measurement of uterine height, abdominal circumference, weight, fetal movement, and blood pressure can be done at home or a nearby community hospital. These measures are agreed with 
recommendations given by specialists from Sichuan province $^{7}$. Articles and courses about self-monitoring, pregnancy management, and check-up plan should be provided online. However, more frequent visits are required when there are pregnancy complications (such as gemellary pregnancy, pregnancy-induced hypertension, intrahepatic cholestasis during pregnancy, etc.).

\section{Admission procedure for pregnant patients}

Outpatient patients to the obstetrics department should make an appointment online (or by phone) and consult a obstetrician before admission, obstetricians should assess their clinical manifestations and risks by online consultation to reduce unnecessary hospital admissions. Pre-examination and triage staff are asked to screen epidemiological history, record symptoms, and test the temperature for all patients visiting hospitals. Patients with positive epidemiological history, fever, cough, or other respiratory symptoms should be directly transferred to the specific fever clinic (through special channels aforementioned) and assessed by diagnostic criteria ${ }^{8}$. For patients who need hospitalization, epidemiological and clinical assessments need to be repeated by obstetricians to pass the COVID-19 screening process, unless it is an emergency situation $^{9}$. The detailed admission procedures are shown in Figure 1. After admission to hospital, only one caregiver is allowed and asked not to leave the hospital during hospitalization. Other people are not allowed to enter the obstetrics department without permission. Respiratory symptoms and body temperature need to be monitored daily. A blood routine examination is advised for all pregnant patients. Patient education about how to wash hands, wear a mask, and cough are emphasized again after admission.

Patients who need hospitalization but whose possibility of infection is not ruled out yet should be isolated in a single bed ward (zone 1 or zone 2). Viral nucleic acid test (nasopharyngeal swab, RT-PCR) and CT scans (CT scans is just for patients from zone 2) need to be done for further confirmation.

Emergency patients (such as vaginal bleeding, premature rupture of membranes, parturition, fetal distress) could be referred directly to the obstetrics department. The COVID-19 screening process needs to be finished after admission. Before ruling out the infection, patients should be treated as a suspected case. In some cases, delivery or surgery needs to be performed immediately before ruling out the infection, then the special isolation delivery or operating rooms are needed. If possible, aerosol-generating procedures, such as general anesthesia with tracheal intubation, should be avoided during surgeries. All recyclable devices should be sterilized after contact with such cases, and standardized protection should be performed just like it is done for the confirmed patients.

\section{Management of childbirth of suspected/con-} firmed patients

The special isolation delivery room (operating room) equipped with newborn rescue equipment (including neonatal incubator) is needed for suspected/confirmed patients, and the distance between

\section{FIGURE 1}

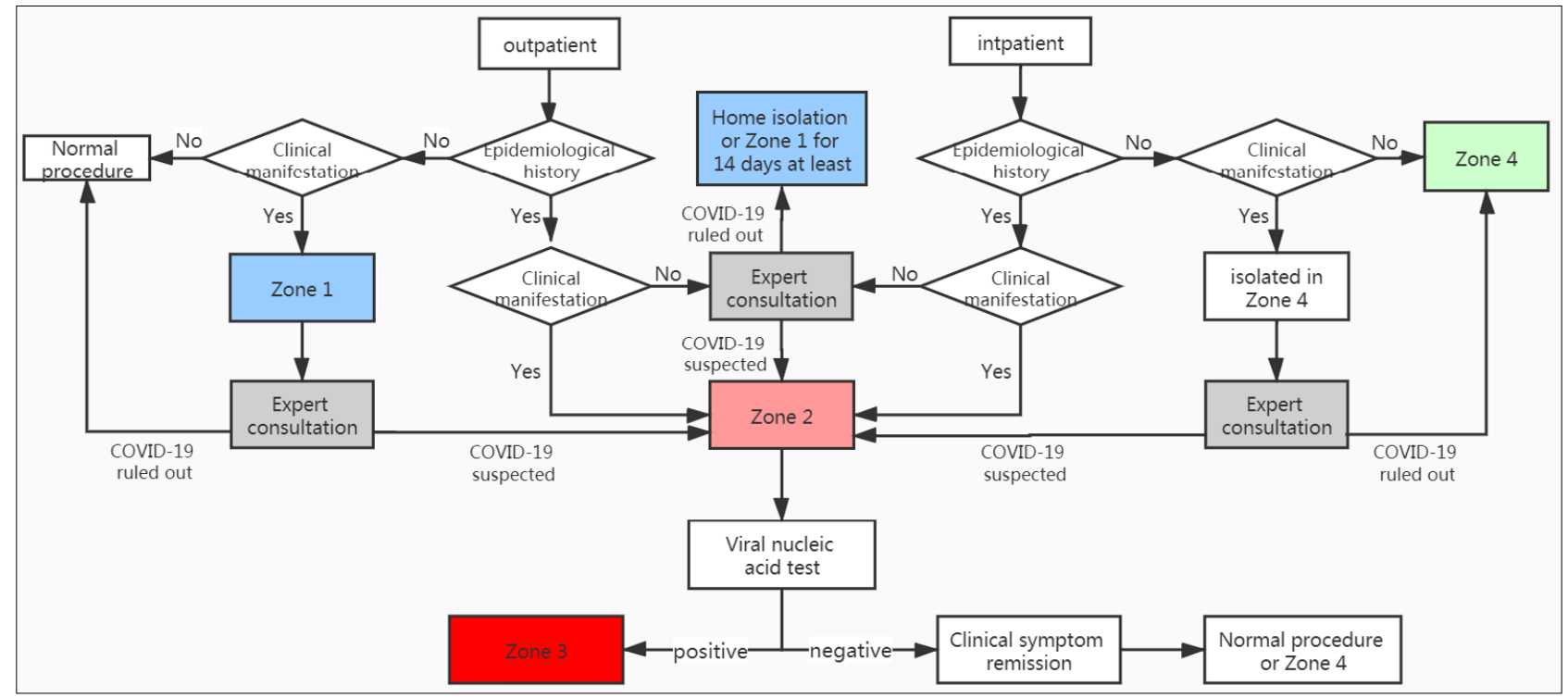


the delivery bed and newborn rescue equipment should be 2 meters at least. A plan for the intrahospital transport channel (elevator) should be made in advance. If possible consumable medical material should be used. At the time of delivery, the obstetrician should notify the newborn pediatrician to come to the operating room (delivery room) in advance to ensure that there is sufficient time for the newborn pediatrician to complete protective preparations. Medical personnel should strictly implement the hand hygiene and three-level protective measures (disposable cap, protective face shield or positive pressure headgear, goggles, medical protective mask, disposable coverall protective clothing, medical rubber gloves, and disposable shoe covers). Doctors should cut the umbilical cord as soon as possible after delivery and reduce close contact with the mother. After delivery, the puerpera should be transferred to the quarantine ward. Neonates would be quarantined in the neonatal quarantine observation ward and transported using a special neonatal incubator. Breastfeeding should be postponed until the possibility of infection is ruled out or the infection is cured. The neonatal quarantine observation period is recommended to be more than 14 days. All reusable devices, operating room (delivery room), and intrahospital transport channel should be sterilized after contact with such patients, and all medical and domestic waste should be disposed of as infectious waste.

On the basis of this management strategy, no SARS-CoV-2 infections happened in our obstetrics departments. However, the success of this strategy depends on sufficient medical resources and healthcare settings to some extent. Our recommendations should be updated continuously with accumulated clinical evidence and the increase in knowledge about COVID-19 over time.

\section{Author's Contribution}

All the authors conform with the International Committee of Medical Journal Editors (ICMJE) criteria for authorship, contributed to the intellectual content of the paper, and gave approval for the final version. Bo Wu, Ying Pu, Han Wang contributed to the writing and editing of the paper.

\section{Conflict of interest}

The authors declare there are no conflicts of interest.

There was no funding.

\section{REFERENCES}

1. Khachfe HH, Chahrour M, Sammouri J, Salhab H, Makki BE, Fares M. An epidemiological study on COVID-19: a rapidly spreading disease. Cureus. 2020;12(3):e7313.

2. Johns Hopkins University. 2019 novel coronavirus COVID-19 (2019-nCoV) data repository by Johns Hopkins CSSE. [cited 2020 May 20]. Available from: https://www.arcgis.com/apps/opsdashboard/index.html\#/ bda7594740fd40299423467b48e9ecf6

3. Poon LC, Yang H, Kapur A, Melamed N, Dao B, Divakar H, et al. Global interim guidance on coronavirus disease 2019 (COVID-19) during pregnancy and puerperium from FIGO and allied partners: information for healthcare professionals. Int J Gynaecol Obstet. 2020;149(3):273-86.

4. Siston AM, Rasmussen SA, Honein MA, Fry AM, Seib K, Callaghan WM et al; Pandemic H1N1 Influenza in Pregnancy Working Group. Pandemic 2009 influenza $A$ (H1N1) virus illness among pregnant women in the United States. JAMA. 2010;303(15):1517-25.
5. Wong SF, Chow KM, Leung TN, Ng WF, Ng TK, Shek CC, et al. Pregnancy and perinatal outcomes of women with severe acute respiratory syndrome. Am | Obstet Gynecol. 2004;191(1):292-7.

6. Zaigham M, Andersson O. Maternal and perinatal outcomes with COVID19: a systematic review of 108 pregnancies. Acta Obstet Gynecol Scand. 2020. doi: 10.1111/aogs.13867.

7. Lv B, Chen M, Liu H. Pregnancy management during the outbreak of novel coronavirus. J Prac Obst Gynecol. 2020;36(2):101-3.

8. National Health Commission of the People's Republic of China. Diagnosis and treatment plan of corona virus disease 2019 (tentative sixth edition). Glob Health J. 2020;10.1016/j.glohj.2020.03.001. doi:10.1016/j. glohj.2020.03.001

9. Chen $Y, L i Z$, Zhang YY, Zhao WH, Yu ZY. Maternal health care management during the outbreak of coronavirus disease 2019. J Med Virol. 2020. doi: 10.1002/jmv.25787. 